\title{
Helper T-cell heterogeneity: a complex developmental issue in the immune system
}

\author{
Cellular \& Molecular Immunology (2010) 7, 163; doi:10.1038/cmi.2010.23
}

A fter activation by antigen-presenting cells, naive, antigenspecific $\mathrm{CD}^{+} \mathrm{T}$ cells differentiate into effector $\mathrm{T}$ cells. Two decades ago, Coffman and Mosman first discovered the heterogeneity of effector T cells, which were named as Th1 or Th 2 cells. ${ }^{1}$ Th1 and Th2 cells are differentially induced and are involved in immunity against intracellular and extracellular pathogens, respectively, as well as immunopathologies such as autoimmunity and allergy. The Th1/ Th2 dichotomy dominated the field of immune regulation until about 4 years ago when IL-17-expressing T cells were proposed to be a third lineage of helper T cells. ${ }^{2,3}$ In addition to these so-called Th17 cells, additional T-cell subsets were also discovered or studied, including Foxp ${ }^{+}$regulatory $\mathrm{T}$ (Treg) cells, $\mathrm{T}$ follicular helper (Tfh) cells and IL-9-expressing 'Th9' cells.

In this issue, experts offer timely summaries of the development and function of novel T-cells subsets. Here I discuss several issues that are raised in these reviews and provide a personal perspective.

Subset or lineage: The heterogeneous phenotypes of Th cells can be termed as states, subsets and lineages. In my view, the term 'lineage' should be defined stringently, i.e., meaning an independent, genetically controlled cell differentiation pathway. For example, it is widely accepted that Th1, Th2, Th17 and Treg cells are of different lineages. They are generated independently during immune responses. Also 'master' transcription factors have been found to mediate their differentiation. Small non-coding RNAs may also emerge as key players in this process, as discussed by Wei and Pei in this issue. ${ }^{4}$ Wan reviews the differentiation of Treg cells ${ }^{5}$ and Xu et al. reviews the differentiation of Th17 cells. ${ }^{6}$ Tfh cells may add to Th list with the discovery of Bcl6 as its key transcriptional regulator, which is covered by $\mathrm{Yu}$ et $a l^{7}$ and Nurieva et al. ${ }^{8}$ Cells that are differentiated into the same pathway may also exhibit heterogeneity. This can be stochastic or the result of different environmental cues.

Flexibility or plasticity: Th1 and Th2 cells were originally believed to be exclusive. However, recent studies on Th17, Treg and Tfh cells have revealed greater flexibility of T-cell phenotypes. $\mathrm{T}$ cells in the central nervous systems of mice in experimental autoimmune encephalomyelitis experiments sometimes coexpress IL-17 and interferon$\gamma$ or IL-17 and Foxp3. This degree of flexibility may allow T cells to have dual function or regulation, which may be important for the function of Treg and Tfh cells at least. Another level of complexity has been documented that effector $\mathrm{T}$ cells can switch their genetic program and become another type. This plasticity has been observed in Treg and Th17 cells. However, questions remain about the physiological and pathological consequences of T-cell plasticity, which require further research to be better understood in the future.

Protective or pathogenic: Diverse adaptive immunity, characterized in part by the distinct effector T cells, is induced by innate responses to various environmental pathogens. It is clear that the additional Th subsets that have been discovered, such as Th17 and Tfh cells, offer protection to the host against the infection, as described by Xu et al. ${ }^{6}$ While the immune system is powerful against foreign pathogens, unbalanced or unwanted immune responses may result in autoimmunity. Leung et al. discuss the interplays of pathogenic Th1 and Th17 cells and anti-inflammatory Treg cells in autoimmunity. ${ }^{9}$ Enhanced Tfh function is also associated with certain systemic autoimmune diseases. ${ }^{8}$ It is important to consider strategies to shift the equation into protection from pathogenesis in patients.

In summary, this series of reviews highlights the recent developments in T-cell subset research. They present the current understanding of the regulation and function of $\mathrm{T}$ cells in immunity and immune diseases, and may guide best practice in basic, translational and clinical research.

Chen Dong
Department of Immunology and Center for Inflammation and Cancer,
MD Anderson Cancer CenterHouston, TX, USA
E-mail: cdong@mdanderson.org

1 Mosmann TR, Coffman RL. TH1 and TH2 cells: different patterns of lymphokine secretion lead to different functional properties. Annu Rev Immunol 1989; 7: 145-173.

2 Harrington LE, Hatton RD, Mangan PR, Turner H, Murphy TL, Murphy KM et al. Interleukin 17-producing $\mathrm{CD}^{+}{ }^{+}$effector T cells develop via a lineage distinct from the Thelper type 1 and 2 lineages. Nat Immunol 2005; 6: 1123-1132.

3 Park H, Li Z, Yang XO, Chang SH, Nurieva R, Wang YH et al. A distinct lineage of CD4 T cells regulates tissue inflammation by producing interleukin 17. Nat Immunol 2005; 6: 1133-1141.

4 Wei B, Pei G. MicroRNAs: critical regulators in Th17 cells and players in diseases. Cell Mol Immunol 2010; 7: 175-181.

5 Wan YY. Regulatory T cells - immune suppression and beyond. Cell Mol Immunol 2010; 7: 204-210.

6 Xu S, Cao X. Interleukin-17 and its expanding biological functions. Cell Mol Immunol 2010; 7: 164-174.

7 Yu D, Vinuesa CG. Multiple checkpoints keep follicular helper T cells under control to prevent autoimmunity. Cell Mol Immunol 2010; 7: 198-203.

8 Nurieva R, Chung $Y$. Understanding the development and function of $T$ follicular helper cells. Cell Mol Immunol 2010; 7: 190-197.

9 Leung S, Liu X, Fang L, Chen X, Guo T, Zhang J. Cytokine milieu in interplay of pathogenic TH1/TH17 cells and regulatory T cells in autoimmune disease. Cell Mol Immunol 2010; 7: 182-189. 